\title{
Partial protective effect of rutin on multiple low dose streptozotocin-induced diabetes in mice
}

Type 1 diabetes or insulin-dependent diabetes mellitus (IDDM) is an autoimmune disorder affecting approximately 5.3 million people (mostly the children or young adults) worldwide, which accounts for 5 to $10 \%$ of all diagnosed cases of diabetes. ${ }^{[1]}$ It is characterized by selective immune cellmediated destruction of pancreatic beta islets, followed by absolute insulin deficiency and chronic hyperglycemia. There is interplay of various cytokines secreted by infiltrating cells into islets (insulitis) and free radicals, destructing pancreatic beta islets. ${ }^{[1,2]}$ In view of the severity of the disease and lack of curative therapy for patients with diabetes (who have to depend only on insulin for survival), many attempts have been made to prevent type 1 diabetes in susceptible individuals. Owing to the problems such as toxicity and adverse reactions associated with certain synthetic agents (immunosuppressants, specific inhibitors of proinflammatory cytokines, and antioxidants), ${ }^{[2-4]}$ a major effort is currently underway to look for compounds from the so-called safer natural products for the prevention of type 1 diabetes. Rutin is a well-known flavonoid found in many plants, is used clinically for the treatment of venous insufficiency/varicose veins, and is generally well tolerated. ${ }^{[5,6]}$ It is reported to have antithrombotic, antiinflammatory, antioxidant, anticarcinogenic, cytoprotective, hepatoprotective, vasoprotective, smooth muscle relaxing, and tissue protein glycation inhibiting activities. ${ }^{[5-8]}$ Its antioxidant activity is proposed to account, in part, for many of its activities..$^{[5,7]}$ Because the oxidative stress predominantly participates in the pancreatic beta cell injury and subsequent development of diabetes, ${ }^{[2]}$ the present study was undertaken to see whether the antioxidant, rutin would protect against the development of diabetes in multiple low dose streptozotocin (MLDS)-injected mice, a model of autoimmune type 1 diabetes.

Type 1 MLDS diabetes was induced in adult male Swiss albino mice (28-30 g) by multiple intraperitoneal (i.p.) injection of subdiabetogenic doses of streptozotocin (STZ; Sigma Chemicals, MO, USA) (40 mg/kg, body weight) once daily for 5 consecutive days, whereas the control rats were given the vehicle citrate buffer ( $\mathrm{pH} 4.4$ ) as per the literature methodology. ${ }^{[4]}$ The dose of rutin $(200 \mathrm{mg} / \mathrm{kg} \mathrm{b.w.)}$ was selected on the basis of the previous literature ${ }^{[7]}$ and prepared as a suspension with $1 \%$ sodium carboxymethyl cellulose (Na-CMC; LOBA CHEMIE, Mumbai) at a dose volume $10 \mathrm{~mL} / \mathrm{kg}$, b.w. There were four experimental groups consisting of 10 mice each. Group I (normal control) and Group II (normal treated) received vehicle $1 \% \mathrm{Na}-\mathrm{CMC}(10 \mathrm{ml} / \mathrm{kg})$ or rutin $(200 \mathrm{mg} / \mathrm{kg})$ once daily for 14 days before and then continuously for the next 7 days after the first vehicle (citrate buffer) injection, respectively. Likewise, Group III (MLDS control) and IV (MLDS treated) received the respective vehicle or rutin treatment before and after the first STZ injection as above. The date of first STZ injection is considered as day 1.

Blood was collected from the retro-orbital sinus of mice under light ether (S.D. Fine-Chem Ltd, Mumbai) anesthesia into the eppendorf tubes containing heparin (S.D. Fine-Chem Ltd, Mumbai), before the administration of rutin (0 day) and at 7, 14, 21, and 28 days after the first vehicle or STZ injection. The plasma was analyzed for glucose concentration by the glucose oxidase-peroxidase (GOD-POD) method, using commercially available spectrophotometric kit (Accurex Pvt. Ltd, Mumbai). The mice with nonfasting plasma glucose level (PGL) of $\geq 300 \mathrm{mg} / \mathrm{dL}$ were considered diabetic. The mean PGL and prevalence of diabetes (cumulative \% incidence of diabetic mice) at different time intervals were determined in the treated animals as compared to the controls. The protocol of this experiment (IAEC/02/07) was approved by the Institutional Animal Ethics Committee (IAEC). The animals were housed and fed as per the standard guidelines of CPCSEA. The results of the PGL are represented as mean \pm SEM. The data of PGL in different groups were analyzed by one-way ANOVA followed by Tukey's test. The prevalence of diabetes was analyzed using Chi-square test. $\mathrm{P}<0.05$ was considered statistically significant.

The results are illustrated in Figure 1 and 2. The mice treated with MLDS demonstrated a significant $(\mathrm{P}<0.05)$ and progressive rise in PGL attaining peak at twenty-first day after STZ injection as compared to vehicle-treated normal control mice. Upon examination on the twenty-first day of STZ injection, all mice developed diabetes (PGL $\geq 300 \mathrm{mg} / \mathrm{dL}$, Figure 1). Prior chronic administration of rutin reduced the prevalence of diabetes from $60 \%$ to $30 \%$ at day 14 , from $100 \%$ to $60 \%$ at day 21 and 28. The over all difference in the prevalence of diabetes was significant $(\mathrm{P}<0.001)$. Also, rutin significantly but not completely prevented the development of hyperglycemia in MLDS mice as shown by significant $(\mathrm{P}<0.05)$ reduction in PGL at day 21 and 28 (Figure 2). However, rutin per se did not significantly alter the PGL in normal mice ruling out its hypoglycemic activity (Figure 2).

Therefore, the effect of rutin on the development of hyperglycemia might possibly be due to its protective/antioxidant mechanisms either by scavenging free radicals produced 
Figure 1. Effect of rutin on the prevalence of diabetes in multiple low dose streptozotocin-injected (MLDS) mice

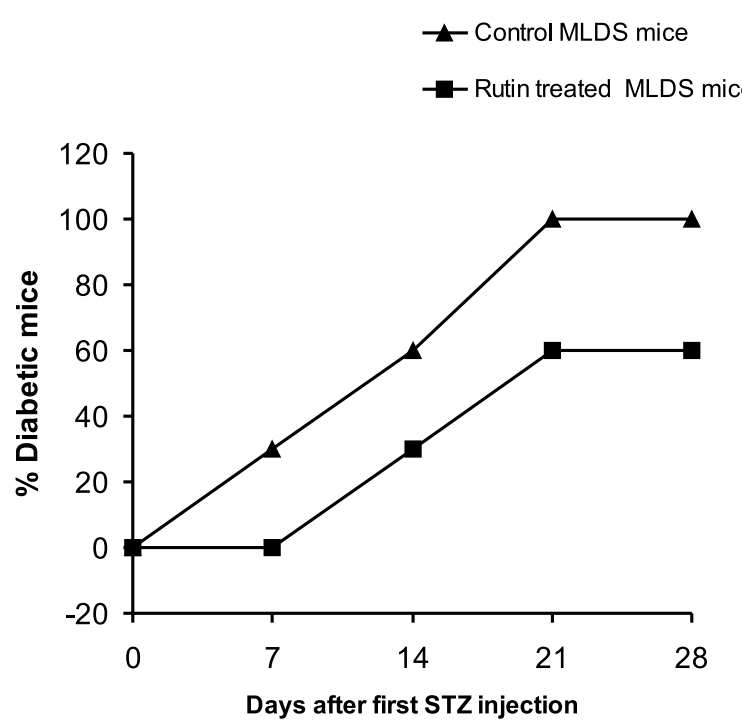

$\mathrm{n}=10$ mice/group. ${ }^{*} \mathrm{P}<0.001$ by Chi-Square test for over all difference in cumulative frequency distribution on incidence of diabetes at different time intervals in rutin treated mice as compared to MLDS control group

by STZ itself and infiltrated inflammatory cells or by increasing the amount of antioxidant levels in beta cells, thereby protecting the insulin-secreting-beta cells from the oxidative damage in MLDS mice. The protective effect of rutin on beta cells by also blocking insulitis process (due to its antiinflammatory action) could not be ruled out and needs further investigation. In conclusion, our study demonstrates for the first time that rutin has partial protective effect against the development of diabetes in MLDS Swiss mice and thus, a potential to exploit it for the prevention of type 1 diabetes in susceptible individuals exist.
Figure 2. Effect of rutin on the plasma glucose level (PGL) in normal and multiple low dose streptozotocin-injected (MLDS) mice

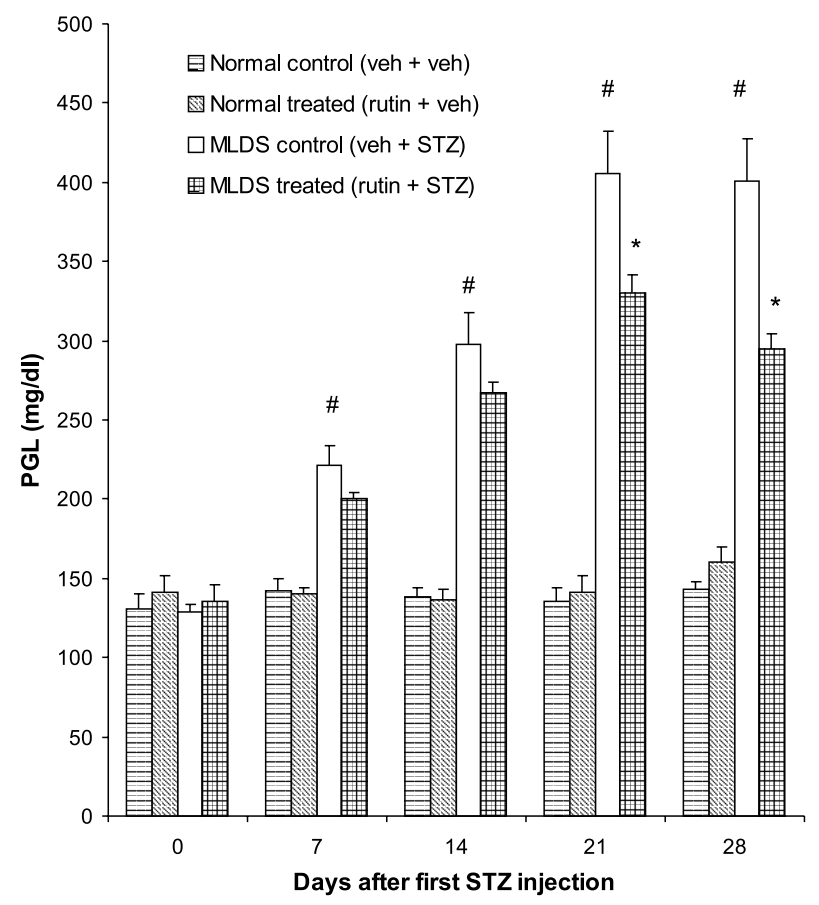

Error bars represent SEM, $\mathrm{n}=10$ in each group. "P<0.05; Vehicle treated normal Vs MLDS control group. ${ }^{*} \mathrm{P}<0.05$; MLDS control Vs MLDS treated group. PGL-plasma glucose level

\section{References}

1. International Diabetes Federation [homepage on the internet]. Available from: http:// www.idf.org/

2. Heineke EW, Johnson MB, Dillberger JE, Robinson KM. Antioxidant MDL 29,311 prevents diabetes in non obese diabetic and multiple low-dose STZ-injected mice. Diabetes 1993;42:1721-30.

3. Nicoletti F, Di Marco R, Morrone S, Zaccone P, Lembo D, Grasso S, et al. Reduction of spontaneous autoimmune diabetes in diabetes-prone BB rats with the novel immunosuppressant fusidic acid. Effect on T-cell proliferation and production of interferon-g. Immunology 1994;81:317-21.

4. Takamura T, Ando H, Nagai Y, Yamashita H, Nohara E, Kobayashi K.-i. Pioglitazone prevents mice from multiple low dose streptozotocin-induced insulitis and diabetes. Diabetes Res Clin Pract 1999;44:107-14.

5. Yildizoglu- Ari N, Altan V, Altinkurt O, Ozturk Y. Pharmacological effects of rutin. Phytother Res 1991;5:19-23.

6. Wadworth AN, Faulds D. Hydroxyethylrutosides. A review of its pharmacology and therapeutic efficacy in venous insufficiency and related disorders. Drugs 1992:44:1013-32.

Department of Pharmacology and Toxicology, National Institute of Pharmaceutical Education and Research (NIPER), Phase X, Sector 67, S.A.S. Nagar, Punjab- 160 062, India. E-mail: ramaraop@yahoo.com
7. La Casa C, Villegas I, Alarcon de la Lastra C, Motilva V, Martin Calero MJ. Evidence for protective and antioxidant properties of rutin, a natural flavone, against ethanol induced gastric lesions. J Ethnopharmacol 2000;71:45-53.

8. Nagasawa T, Tabata N, Ito Y, Aiba Y, Nishizawa N, Kitts DD. Dietary G-rutin suppresses glycation in tissue proteins of streptozotocin-induced diabetic rats. Mol Cell Biochem 2003; 252:141-147. 